\title{
A Molecular Dynamics Study of the Structural and Dynamical Properties of Putative Arsenic Substituted Lipid Bilayers
}

\author{
Hui-Hsu Gavin Tsai ${ }^{1, *}$, Jian-Bin Lee ${ }^{1}$, Jian-Ming Huang ${ }^{1}$ and Ratna Juwita ${ }^{1,2}$ \\ 1 Department of Chemistry, National Central University, Jhong-Li City, Tao-Yuan County 32001, \\ Taiwan; E-Mails: 0920bin@gmail.com (J.-B.L.); casuter023@hotmail.com (J.-M.H.); \\ raju_c4c4@yahoo.co.id (R.J.) \\ 2 Department of Chemistry, Brawijaya University, J1. Veteran, Malang 65145, Indonesia \\ * Author to whom correspondence should be addressed; E-Mail: hhtsai@cc.ncu.edu.tw; \\ Tel.: +886-3-422-7151 (ext. 65909); Fax: +886-3-422-7664.
}

Received: 1 March 2013; in revised form: 23 March 2013 / Accepted: 29 March 2013 /

Published: 9 April 2013

\begin{abstract}
Cell membranes are composed mainly of phospholipids which are in turn, composed of five major chemical elements: carbon, hydrogen, nitrogen, oxygen, and phosphorus. Recent studies have suggested the possibility of sustaining life if the phosphorus is substituted by arsenic. Although this issue is still controversial, it is of interest to investigate the properties of arsenated-lipid bilayers to evaluate this possibility. In this study, we simulated arsenated-lipid, 1-palmitoyl-2-oleoyl-sn-glycero-3-arsenocholine (POAC), lipid bilayers using all-atom molecular dynamics to understand basic structural and dynamical properties, in particular, the differences from analogous 1-palmitoyl-2oleoyl-sn-glycero-3-phosphocholine, (POPC) lipid bilayers. Our simulations showed that POAC lipid bilayers have distinct structural and dynamical properties from those of native POPC lipid bilayers. Relative to POPC lipid bilayers, POAC lipid bilayers have a more compact structure with smaller lateral areas and greater order. The compact structure of POAC lipid bilayers is due to the fact that more inter-lipid salt bridges are formed with arsenate-choline compared to the phosphate-choline of POPC lipid bilayers. These inter-lipid salt bridges bind POAC lipids together and also slow down the head group rotation and lateral diffusion of POAC lipids. Thus, it would be anticipated that POAC and POPC lipid bilayers would have different biological implications.
\end{abstract}

Keywords: arsenic-loving life; arsenated-lipid; lipid bilayers; molecular dynamics 


\section{Introduction}

Phosphorus is one of the six most important chemical elements, in addition to carbon, oxygen, nitrogen, hydrogen, and sulfur, which are essential for all forms of life. Phosphorus is a component of adenosine triphosphate (ATP), a principal energy carrier in the cells of organism. The sugar-phosphate chain forms the structural backbone of DNA and RNA. The phosphate group of phospholipids are a major component of cell membranes. In 2011, Wolfe-Simon and co-workers claimed to have discovered a bacterium strain, GFAJ-1 of the Halomonadaceae, isolated from the arsenic-rich waters of Mono Lake, California, that can use arsenic in place of phosphorus to sustain its growth [1]. While arsenic has some chemical similarities with phosphorus, it is usually toxic to most living things. The GFAJ-1 bacteria however had incorporated arsenic into their DNA and proteins and radiolabeled ${ }^{73} \mathrm{AsO}_{4}{ }^{3-}$ experiments showed a small fraction of ${ }^{73} \mathrm{AsO}_{4}{ }^{3-}$ was also present in the lipids. This study, however, received many controversial comments. One of the comments is that the arsenate compounds would undergo very rapid hydrolytic cleavage in water [2]. In contrast, the phosphodiester linkages of native DNA is stable with a hydrolysis half-life of approximately $30,000,000$ years at $25^{\circ} \mathrm{C}$. Nevertheless, a theoretical functional density study, performed by Gu, Leszczynski, and co-workers [3], revealed that the base-stacking and base-base pairing structure of DNA could increase the stability of arsenate in respect to hydrolysis, in comparison with isolated arsenate model compounds. However, this computational study also suggested that arsenated-DNA is still less stable than normal DNA under hydrolysis.

On the other hand, recent studies revealed that although the bacterial strain GFAJ-1 discovered in California's Mono Lake is arsenic-tolerant, it requires phosphorus [4]. Further, mass spectrometry did not detect covalently bound arsenated-DNA. Another study, by Erb, Kiefer and co-workers, showed that GFAJ-1 can grow at low phosphate concentrations, even with the co-existence of the high concentrations of arsenate [5]. In contrast, GFAJ-1 cannot grow in a phosphorus-depleted $(<0.3 \mu \mathrm{M})$ and arsenate-containing environment. The study of Elia,Wellner and co-workers showed that the "arsenic-life" bacteria strongly prefer phosphorus over arsenic [6]. They tested five types of periplasmic phosphate-binding proteins (PBPs) that can uptake phosphate in bacterial cells. Their results showed that all tested PBPs prefer phosphate to arsenate at least 500-fold; in particular, one of the PBPs of GFAJ-1 shows roughly 4500-fold preference for phosphate over arsenate. This work provides a comprehensive understanding of how GFAJ-1 can grow at a high concentration of arsenate.

Although the issue of existence of arsenic-based life is controversial, it remains an open and interesting research topic [7,8] due to the similar chemical properties of phosphorus and arsenic [9]. Arsenate and phosphate have striking chemical similarities. For examples, the arsenate, AsO43-, has the same tetrahedral structure, bonding sites, and nearly identical $\mathrm{pKa}$ as phosphate, PO43-. Biological membranes, usually present in the form of phospholipid bilayers, play significant roles in controlling the compartment, transportation, and communication functions of cells [10]. The extensive chemical similarity between arsenate and phosphate raises the question: what are the structural and dynamical differences between putative arsenic substituted lipid bilayers and native phospholipid bilayers? The structural and dynamical properties of lipid bilayers will influence its biological implications. In this study, we studied putative 1-palmitoyl-2-oleoyl-sn-glycero-3-arsenocholine (POAC) lipid bilayers in which the phosphorus in 1-palmitoyl-2-oleoyl-sn-glycero-3-phosphocholine lipid (POPC) is substituted by arsenic by all-atom molecular dynamics (MD) simulations. The chemical structure of 
POAC and POPC lipids are displayed in Figure 1. We first developed a force field for the arsenate group in POAC, which is compatible with the CHARMM36 lipid force field. Various structural and dynamical properties of POAC lipid bilayers were calculated; we compared the structural and dynamical properties of POAC lipid bilayers with those of POPC lipid bilayers to understand how the arsenate groups affect the structural and dynamical properties of lipid bilayers.

Figure 1. Chemical structures and labeling of atoms of (a) POAC and (b) POPC lipids.
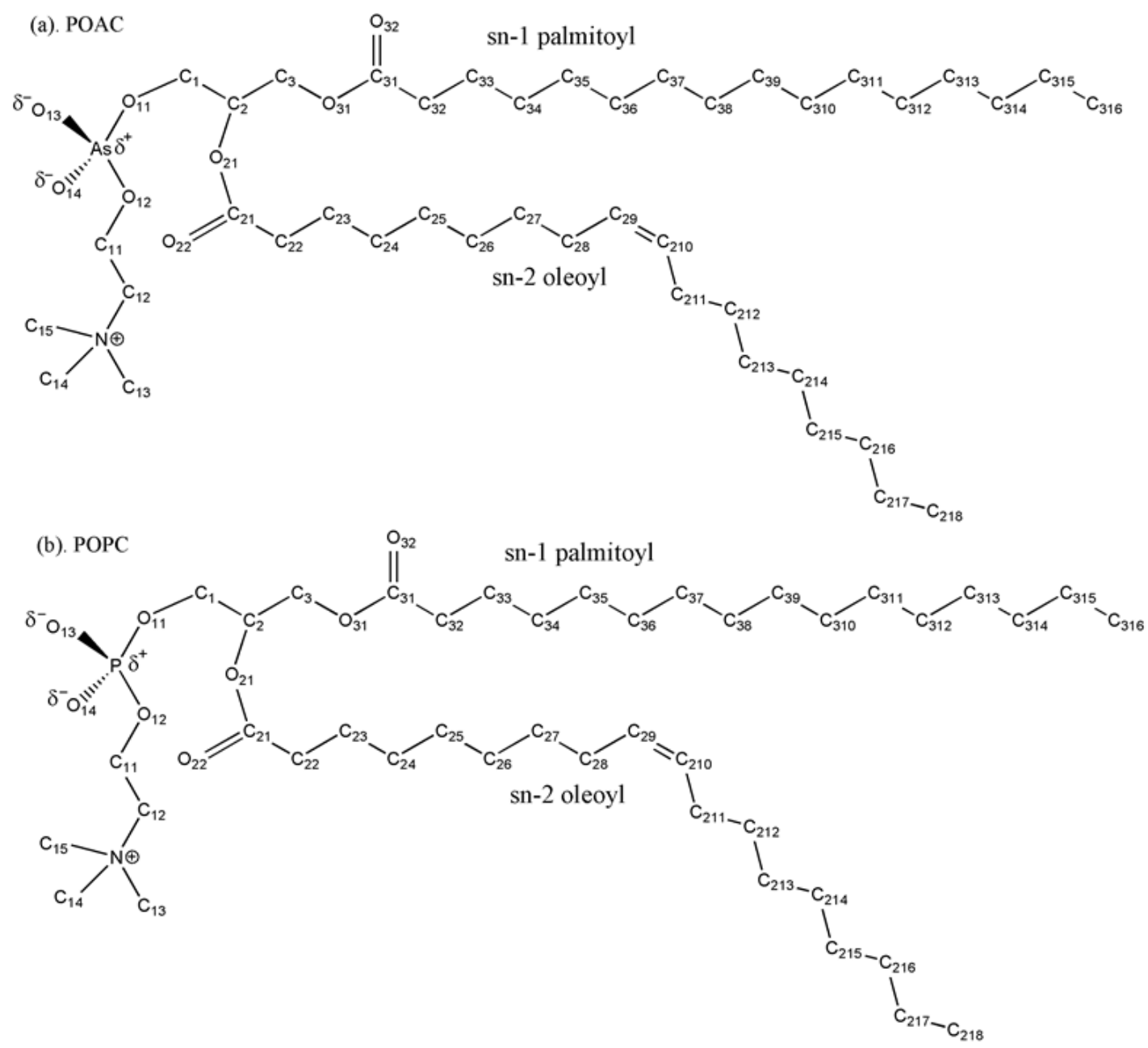

\section{Results and Discussion}

\subsection{Area per Lipid and Membrane Thickness}

First, we used the average area per lipid $<\mathrm{A}>$ as a parameter to determine the equilibrium configurations of the POAC and POPC simulation systems. The value of $<\mathrm{A}>$ was calculated from the lateral surface area of the simulation box divided by the number of lipids in a leaflet. Figure $2 \mathrm{a}$ displays the time evolution of the value of $\angle \mathrm{A}>$ for POAC and POPC lipid bilayers. In the POAC systems, the values of $\angle A>$ decreased rapidly in the first $30 \mathrm{~ns}$, and were stable thereafter. For the POPC simulations, the values of $<A>$ also decreased during the early stage of simulation, and became more stable after approximately 15 ns. Therefore, we used the latter half of the simulation trajectory (75-150 ns) for subsequent statistical analyses. The value of $<\mathrm{A}>$ averaged over $75-150 \mathrm{~ns}$ 
trajectories for the POAC system was $53.25 \pm 1.13 \AA^{2}$, which is $9.57 \AA^{2}$ smaller than that of the POPC system $\left(62.82 \pm 1.22 \AA^{2}\right)$. The experimental values of $<\mathrm{A}>$ in POPC bilayers are in a range from 54 to $68.3 \AA^{2}$ [11-14]. Our calculated result of $<\mathrm{A}>$ is consistent with the experimental values. We also calculated the average membrane thickness $\langle\mathrm{T}\rangle$ in terms of the shortest distance between the phosphorus atom of one lipid in a given leaflet and all of the phosphorus atoms of the lipids in the other leaflet. The values of $\langle\mathrm{T}\rangle$ were averaged over the membrane thickness defined by all of the lipids in a given leaflet. Figure $2 \mathrm{~b}$ displays the time evolution of the value of $\langle\mathrm{T}\rangle$ for POAC and POPC lipid bilayers, which are stable during the time course $75-150 \mathrm{~ns}$. The value of $<\mathrm{T}>$ averaged over $75-150 \mathrm{~ns}$ trajectories for the POAC system was $44.79 \pm 0.77 \AA$, which is $5.34 \AA$ thicker than that of the POPC system $(39.45 \pm 0.65 \AA)$. Our simulated value of $<\mathrm{T}>$ in POPC bilayers is in good agreement with the experimental value of $37.0 \AA$ [13]. Poger and Mark have performed delicate studies of structural and dynamical properties of various lipid bilayers $[15,16]$; they estimated the $<\mathrm{A}>$ and $<\mathrm{T}>$ values of POPC lipid bilayers are $63.8 \AA^{2}$ and $34.6 \AA$, respectively. Our estimated $<\mathrm{A}>$ and $<\mathrm{T}>$ values of POPC lipid bilayers are in good agreement with experimental and simulation results. It is observed that the values of $<\mathrm{T}>$ and $<\mathrm{A}>$ were generally anti-correlated (Figure 2); this relationship might be understood by considering that when the lipid bilayer lateral area is contracted, its head groups are pushed toward the water phase, resulting in a greater membrane thickness, and vice versa.

Figure 2. (a) The average area per lipid $<\mathrm{A}>$ plotted with respect to simulation time for POAC and POPC systems; (b) The average membrane thickness $<\mathrm{T}>$ plotted with respect to simulation time for POAC and POPC systems. Heavy lines are the average of a $5 \mathrm{~ns}$ time window of simulated trajectories.
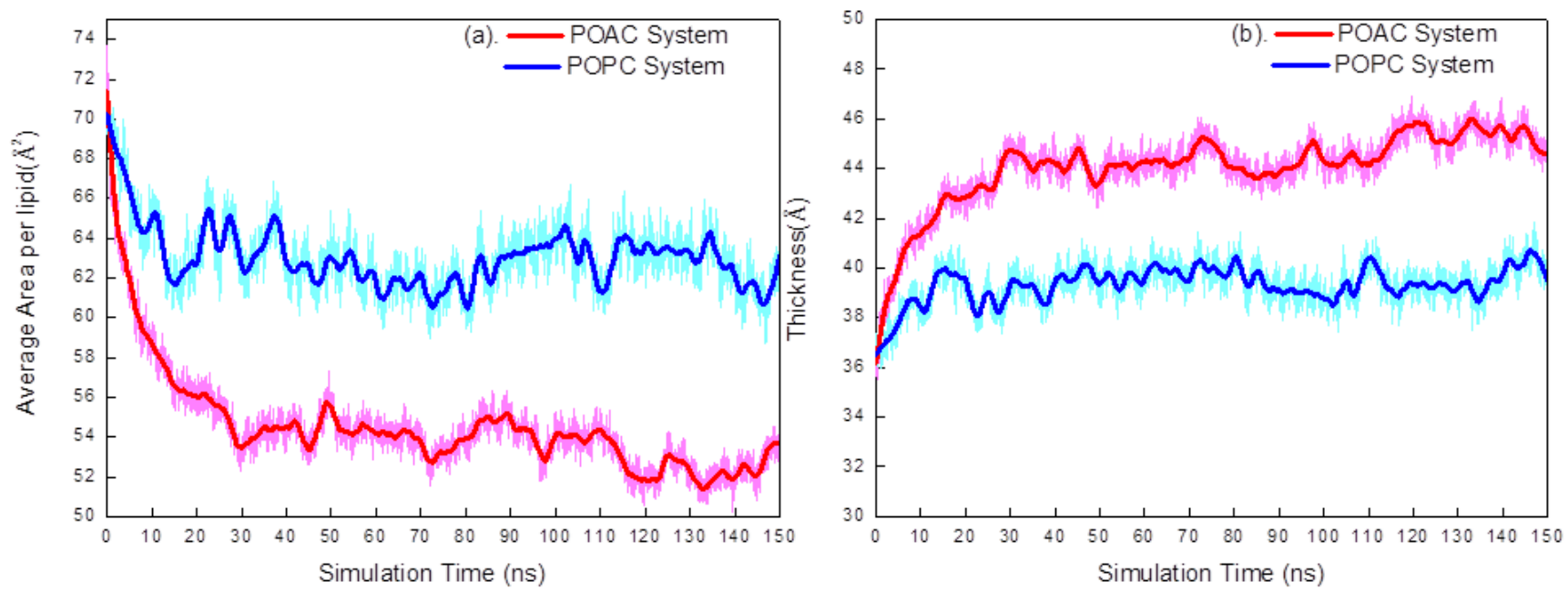

\subsection{Atom Distribution}

To investigate the structures of POAC and POPC lipid bilayers, we calculated the locations of the lipid head group and carbonyl group atoms (arsenate group contains As, O11, O12, O13, and O14 atoms; phosphate contains $\mathrm{P}, \mathrm{O} 11, \mathrm{O} 12, \mathrm{O} 13$, and $\mathrm{O} 14$ atoms; and choline group contains $\mathrm{N}, \mathrm{C} 12$, $\mathrm{O} 13, \mathrm{C} 14$, and $\mathrm{C} 15$ atoms), carbonyl group (C21, O22, C31, and $\mathrm{O} 32$ atoms), the ions, and the water molecules from their corresponding lipid bilayers centers (Figure 3). It is observed that the head groups of the POAC system protruded more toward the water phase with respect to those of POPC 
system. This result is consistent with the calculated membrane thicknesses discussed above: the POAC lipid bilayers were thicker than the POPC system.

Figure 3. Selected atom distributions of (a) POAC lipid bilayers and (b) POPC lipid bilayers.

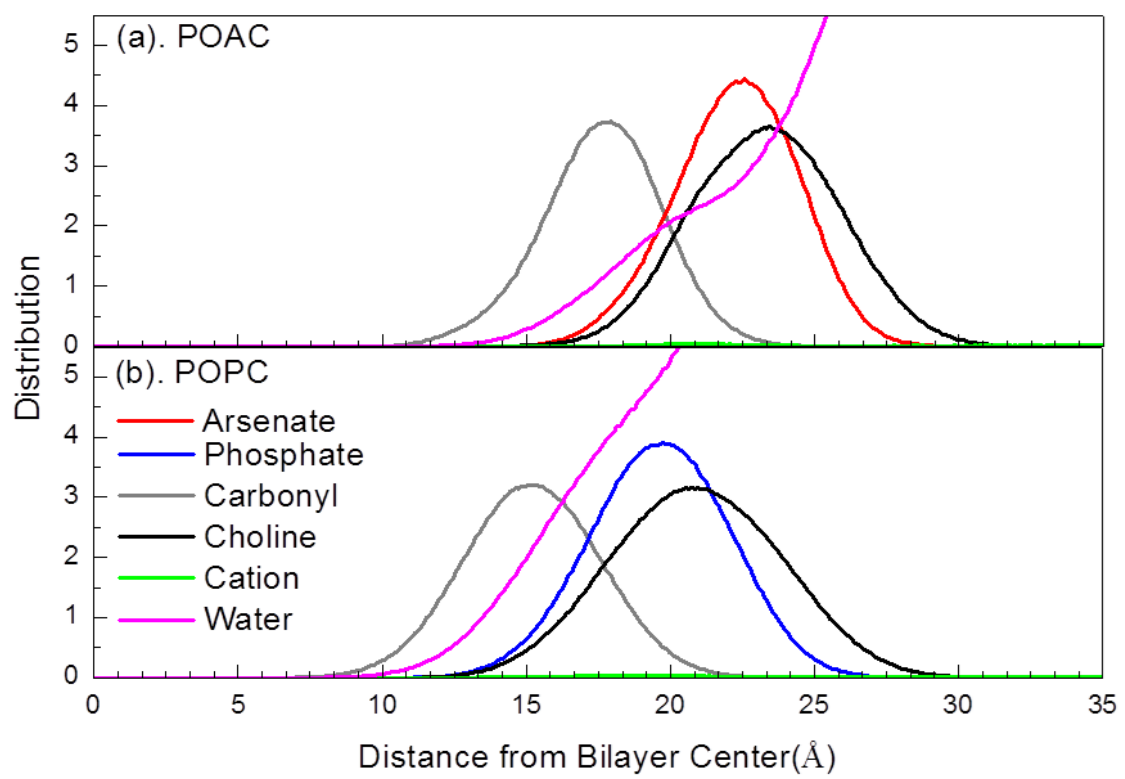

In Figure 3, fewer water molecules were associated with the head groups of POAC lipid bilayers relative to that of POPC lipid bilayers. To determine the hydration level of POAC and POPC lipid bilayers, we calculated the radial distribution functions $g(r)$ for the water molecules (oxygen atoms) with respect to the phosphorus or arsenic atoms (Figure 4a). The $g(r)$ is calculated by

$$
\mathrm{g}(r)=\frac{N(r)}{4 \pi r^{2} \rho \delta r}
$$

where $N(r)$ is the number of two chosen atoms at a distance $r, \delta r$ is a spherical shell of thickness at a distance $r$ of two chosen atoms, and $\rho$ is the number density. It is observed that the POAC lipids were less hydrated than POPC lipids. The average number of water molecules solvated with one lipid, $<\mathrm{H}>$, is 11.46 for the POAC system, while it is 15.06 for the POPC system. The $<\mathrm{H}>$ was calculated with a cutoff distance of $4.85 \AA$, which covered the first peak of Figure $4 \mathrm{a}$.

To further clarify interactions of inter-lipid arsenate/phosphate-choline groups, we calculated the $g(r)$ for the nitrogen atoms on choline groups with respect to the phosphorus or arsenic atoms (Figure $4 b$ ). The distance of the $g(r)$ peak for the POAC system is larger than that of POPC system. This result may occur due to the longer bond lengths in arsenate groups than those of the corresponding ones in phosphate groups. The average number of inter-lipid arsenate (phosphate)-choline salt bridges is 4.94 for the POAC system and 3.15 for the corresponding average number of inter-lipid phosphate-choline salt bridge of POPC system; the POAC lipids forms more inter-lipid arsenate-choline salt bridges than the inter-lipid phosphate-choline salt bridge of POPC systems. This result may be due to the higher electrostatic nature of the oxygen atoms of arsenate compared to those of phosphate. The average number of inter-lipid phosphate-choline salt bridges was calculated with a cutoff distance of $6.75 \AA$, which covered the first peak of Figure $4 \mathrm{~b}$. 
Figure 4. (a) Radial distribution functions $g(r)$ of the nitrogen atom of choline groups with respect to water molecules (oxygen atoms); (b) Radial distribution functions $g(r)$ of the nitrogen atom of choline groups with respect to the arsenic atom of POAC lipids and the phosphorus atom of POPC lipids.

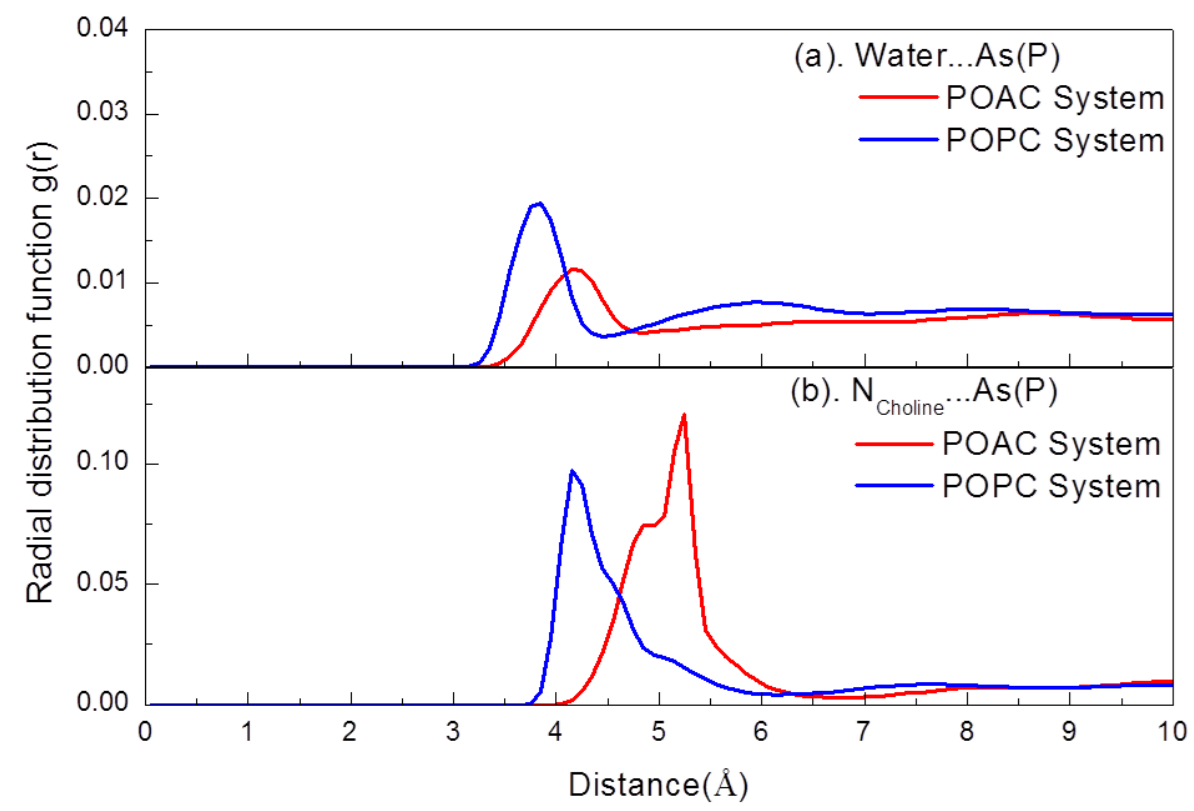

\subsection{Order Parameters}

We calculated the deuterium order parameter of the lipid's acyl chains by the function,

$$
\mathrm{S}_{\mathrm{CD}}=\frac{1}{2}\left\langle 3 \cos ^{2}\left(\theta_{i}\right)-1\right\rangle
$$

where $\theta_{i}$ is the instantaneous angle between the $i$ th segmental vector of the carbon atoms of the acyl chain and the normal of lipid bilayers. The symbol \langle\rangle denotes the average over the selected ensembles and simulation time. Figure 5 presents the values of $S_{\mathrm{CD}}$ of the POAC and POPC systems as well as the experimental values for POPC lipids [17]. For the $s n-1$ chain, the atom C31 is labeled as carbon 1 , the atom C32 is labeled as carbon 2, etc.; for the $s n-2$ chain, the atom C21 is labeled as carbon 1, the atom C22 is labeled as carbon 2, etc. It is seen that the calculated order parameters of POPC lipids are in good agreement with those of experimental values [17]. For a given lipid bilayer, the $s n-1$ chain is more ordered than the $s n-2$ chain. Interestingly, the acyl chains of POAC lipid bilayers are more ordered than those of the POPC system. The result was anti-correlated with the values of $\langle\mathrm{A}\rangle$; it can be understood by that the POAC system with a lower value of $<\mathrm{A}>$ would be more compact than the POAC system and, thus, would pack its acyl chains together, resulting in a more-ordered structure. 
Figure 5. Deuterium order parameters $\mathrm{S}_{\mathrm{CD}}$ of the (a) sn-1 and (b) sn-2 chains of POAC and POPC lipid bilayers.

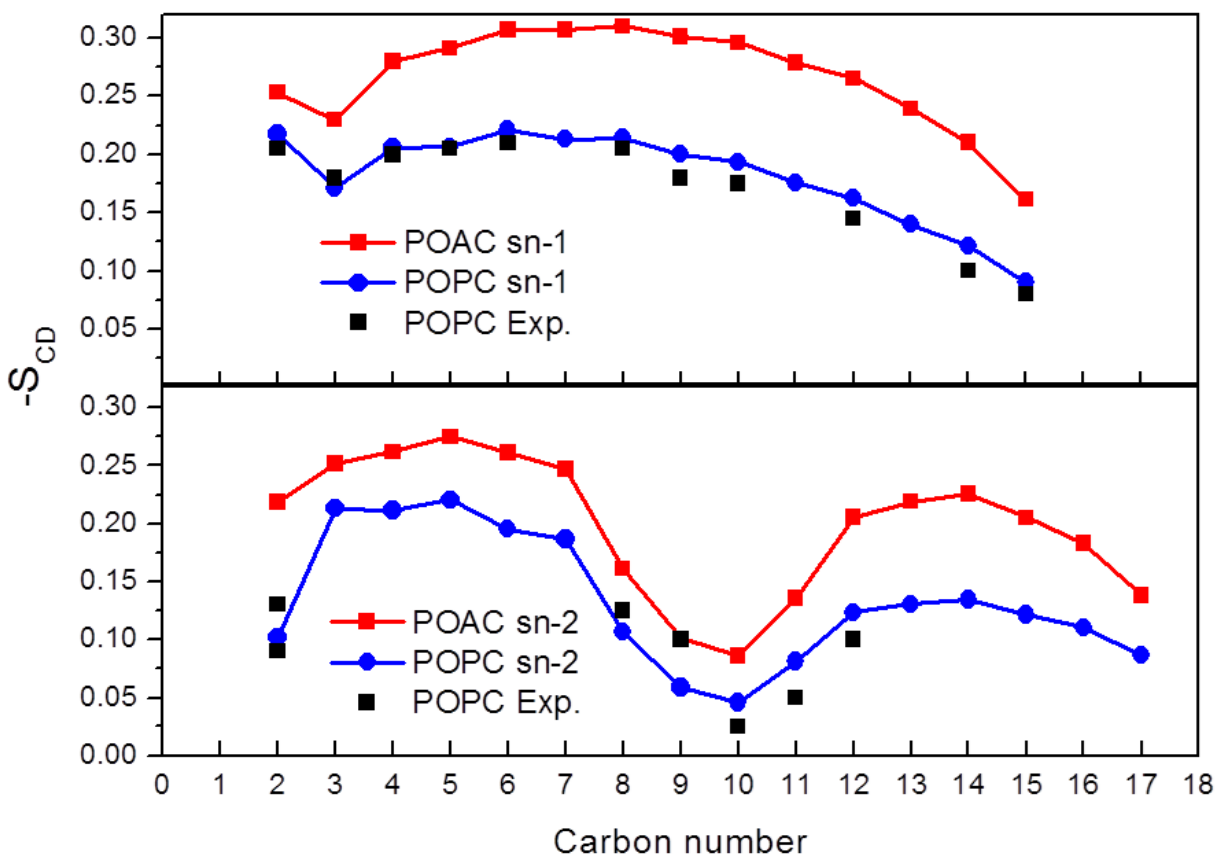

\subsection{Dynamics of Lipids}

We studied the head group dynamics by rotational autocorrelation function (RAF) of the $\mathrm{P} \rightarrow \mathrm{N}$ or $\mathrm{As} \rightarrow \mathrm{N}$ vector to the normal of lipid bilayers (Figure $6 \mathrm{a}$ ) using the equation.

$$
\operatorname{RAF}(t)=\left\langle\bar{v}_{P \rightarrow N}\left(t_{o}\right)_{i} \cdot \overrightarrow{v_{P \rightarrow N}}\left(t_{o}+t\right)_{i}>=\frac{1}{N} \sum_{i=1}^{N} \frac{1}{t_{\max }} \sum_{t_{o}}^{t_{\text {max }}} \overrightarrow{v_{P \rightarrow N}}\left(t_{o}\right)_{i} \cdot \overrightarrow{v_{P \rightarrow N}}\left(t_{o}+t\right)_{i}\right.
$$

where $N$ is the total number of lipid chains and $t_{\max }$ is half of the analysis time (to ensure that the RAF results were calculated from the same amount of data). It is observed that the head group rotation of the POAC lipids is slower than that of the POPC lipids. The average angle (in degrees) of the As $\rightarrow \mathrm{N}$ $\left(<\theta_{\mathrm{As} \rightarrow \mathrm{N}}>\right)$ vector of POAC lipid head group to the normal of lipid bilayers is $75.6^{\circ} \pm 26.7^{\circ}$, which is larger than that of POPC lipids $\left(66.7^{\circ} \pm 27.3^{\circ}\right)$. To obtain the relaxation time $(\tau)$ of head group rotation of lipids, the RAF results were fitted to the Kohlrausch-Williams-Watts (KWW) function $[18,19]$,

$$
\operatorname{RAF}(t)=\exp (-t / \tau)^{\beta}
$$

where $0 \leq \beta \leq 1$. Generally, it does not fit the early and late regions of the decay very well. The KWW relaxation parameters are determined from the slope and $t=1 \mathrm{~ns}$ intercept of the $\ln [-\ln \mathrm{RAF}(\mathrm{t})] v s$. ln $t$ plot by a least-square fit (Figure 6b). The $\tau_{\text {RAF }}$ for the POAC lipids is 95.02 ns which is approximately 19 times slower than that of POPC lipids $\left(\tau_{\mathrm{RAF}}=4.99 \mathrm{~ns}\right)$. 
Figure 6. (a) RAFs of the POAC and POPC systems and (b) Relaxation of the RAF(t) for the POAC and POPC systems.
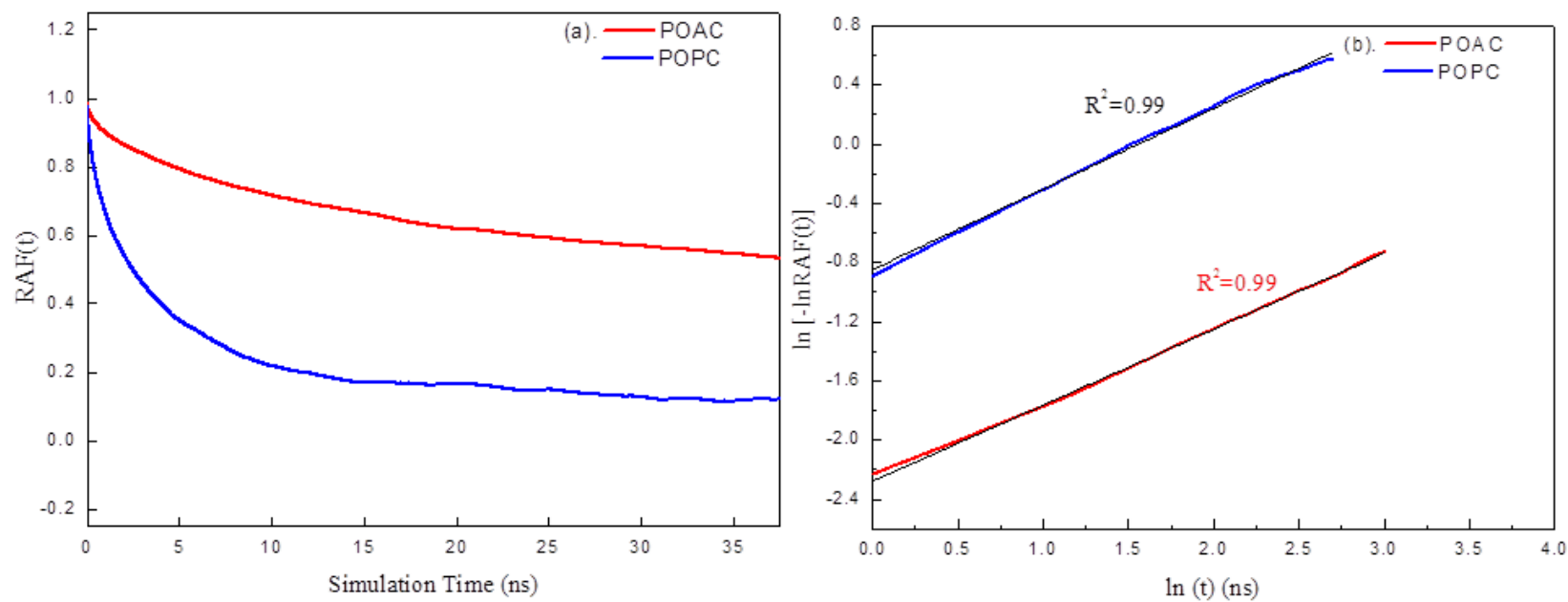

We further calculated the lateral diffusion coefficients of lipids in the $x y$-directions $\left(D_{x y}\right)$ by the mean-square-displacement:

$$
D_{x y}=\frac{1}{4} \lim _{t \rightarrow \infty} \frac{<\left[r\left(t_{o}+t\right)-r\left(t_{o}\right)\right]^{2}>}{t}
$$

where $D_{x y}$ is the lateral self-diffusion coefficient, $r$ is the center of mass of the lipid, and $t$ is the elapsed time. We obtained that the $D_{x y}$ for the POAC lipids is $6.75 \times 10^{-8} \mathrm{~cm}^{2} / \mathrm{s}$, which is slower than that of the POPC lipids $\left(D_{x y}=9.00 \times 10^{-8} \mathrm{~cm}^{2} / \mathrm{s}\right)$.

Taken together, with respect to the corresponding properties of POPC lipid bilayers, our simulations showed that POAC lipid bilayers have smaller lateral areas, greater thicknesses, and greater order. In addition, the lipids of POAC lipid bilayers have slower head group rotation and lateral diffusion coefficients than those of POPC lipid bilayers. These properties are inter-related and can be understood as follows: when the lipid bilayers' lateral area is contracted, the head groups of lipids are pushed toward the water phase, resulting in a greater membrane thickness and at the same time, the lipid will have a greater order of their acyl chains within the compact lipid bilayer matrix. In nature, the lipids will have slower motion within a compact lipid bilayer matrix, which retards the lipids' lateral diffusion and head group rotation. One of the possible reasons for the more compact structure of the POAC lipid bilayers is that more inter-lipid arsenate-choline salt bridges are formed than the corresponding ones of POPC lipid bilayers. These kinds of inter-lipid salt bridges will bind lipids together and retard lipid motion.

The formation of inter-lipid salt bridges may provide additional stabilization, retarding the POAC lipids from being quickly hydrolyzed when they are embedded in their lipid matrix. First, they reduced the water molecules solvated with the arsenate group of POAC lipid. We estimated the average number of water molecules solvated with one lipid is 15.06 for the POPC system, while it is only 11.46 for the POAC system. Second, the inter-lipid salt bridges between POAC lipids may raise the activation energy of POAC hydrolysis by providing a "heavy" leaving group; this effect is similar to the base-stacking in DNA which increases the resistance of As-DNA hydrolysis [3]. The hydrolysis stabilization of POAC lipid bilayers should be higher than that of POAC lipid in its free form. 
The POAC lipid bilayers have distinct structural and dynamics properties that differ from those of POPC lipid bilayers. Therefore, it would be anticipated that POAC lipid bilayers will have different biological implications from native POPC lipid bilayers. However, a study of the biological properties of POAC lipid bilayers is beyond the scope of this work. Nevertheless, since POAC lipid bilayers have a more compact structure, we might anticipate that POAC lipid bilayers will create a higher energy barrier for inter-cellular chemical exchange and protein transportation than that of POPC lipid bilayers.

\section{Computational Methods}

\subsection{Arsenate Force Field Parameterization}

Parameterization of the arsenate group of POAC lipids used the model compounds dimethyl arsenate (DMA, see Figure 7). Parameters of DMA were first developed and then transferred to the dimethyl arsenate group in POAC. To be compatible with the corresponding force fields of POPC, we mainly followed the original methodologies of Karplus et al. [20] in developing the parameters of dimethyl phosphate (DMP). All ab initio calculations were performed by Gaussian 09 program [21]. The structure of DMA was optimized in the gauche-gauche (two O2A-As-OSA-CT3 dihedral angles) conformation at the HF/6-31G* level. The optimized structures of DMA were used to develop a set of 36 internal coordinates and to construct the B matrix, allowing conversion from Cartesian to internal coordinates. The vibrational spectra of DMA was calculated at the HF/6-31G* level based on its optimized structure. A scaling factor 0.9 was applied to scale the vibrational frequencies of DMA. The $\mathrm{U}$ matrix, which permits analysis of the harmonic vibrational frequencies in terms of the potential energy distribution among symmetry-adapted coordinates, was calculated by MOLVIB module implemented in CHARMM program [22].

Figure 7. Water-DMA interaction configurations and atom types.

(a).

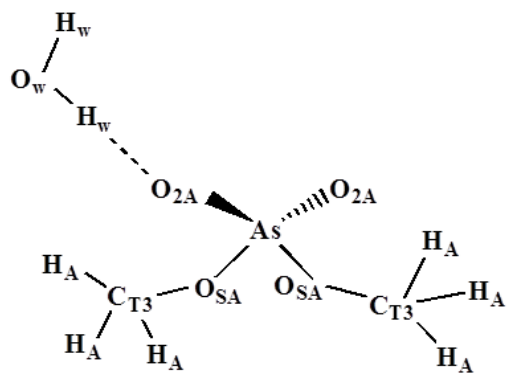

(c).

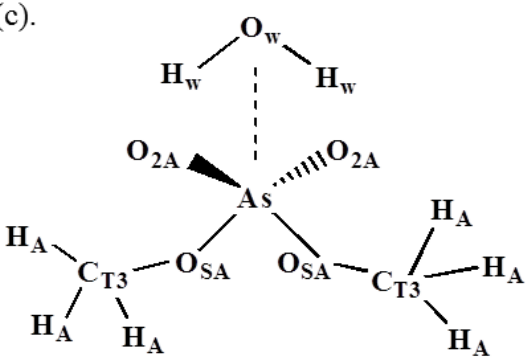

(b).

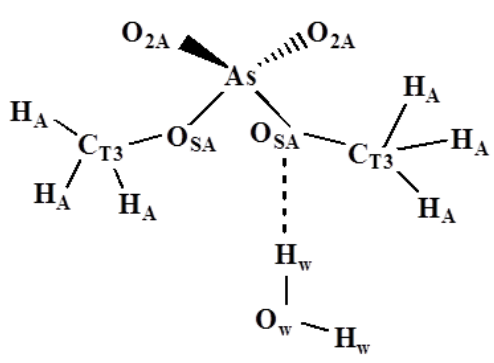

(d).

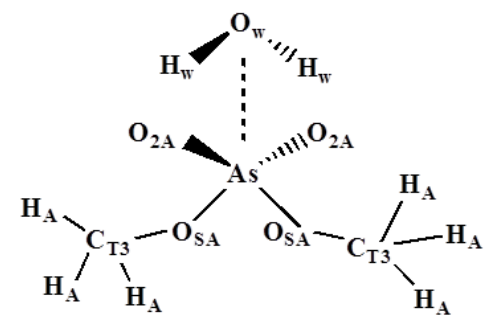


Molecular mechanics were performed using the CHARMM program for which the potential functions were pre-defined. The energy function includes internal and external non-bonded terms; the internal terms include bond stretching, angle bending, and dihedral angle torsion; the external non-bonded interactions are represented by a Lennard-Jones 6-12 term for the van der waals repulsion and dispersion interaction and a Coulomb term for the charge-charge interactions. Their potential functions are expressed as:

$$
\begin{gathered}
\mathrm{U}(\overrightarrow{\mathrm{R}})=\sum_{\text {bonds }} \mathrm{K}_{\mathrm{b}}\left(\mathrm{b}-\mathrm{b}_{0}\right)^{2}+\sum_{\text {angles }} \mathrm{K}_{\theta}\left(\theta-\theta_{0}\right)^{2}+\sum_{\text {dihedrals }} \mathrm{K}_{\phi}(1+\cos (\mathrm{n} \phi-\delta)) \\
+\sum_{\text {nonbond }} \varepsilon\left[\left(\frac{\sigma_{\mathrm{ij}}}{\mathrm{r}_{\mathrm{ij}}}\right)^{12}-2\left(\frac{\sigma_{\mathrm{ij}}}{\mathrm{r}_{\mathrm{ij}}}\right)^{6}\right]+\frac{\mathrm{q}_{\mathrm{i}} \mathrm{q}_{\mathrm{j}}}{4 \pi \mathrm{er}}
\end{gathered}
$$

where $K_{b}, K_{\theta}$, and $K_{\phi}$ are the force constants of bond stretching, angle bending, and dihedral angle torsion, respectively; $b, \theta$, and $\phi$ are the bond length, bond angle, and dihedral angle, respectively; $b_{o}$ and $\theta_{o}$ are the reference bond length and bond angle, respectively. $\varepsilon$ is the Lennard-Jones well-depth and $\sigma$ is the distance at Lennard-Jones minimum, $q_{i}$ is the partial atomic charge and $e$ is the dielectric constant which can depend on $r_{i j}$ and $r_{i j}$ is the distance between atoms $i$ and $j$.

Non-bonded interaction parameters (point charges, $\varepsilon$, and $\sigma_{\mathrm{ij}}$ ) were optimized to maintain a proper balance between solvent-solute interactions of all configurations shown in Figure 7. The solvent-solute interaction energies were calculated from $a b$ initio calculations with the corrections of basis set superposition error [23]. The optimized interaction energy and distance are shown in Table 1, which are good agreement with the $\mathrm{HF} / 6-31 \mathrm{G}^{*}$ values for all configurations. The optimized non-bonded parameters are provided in the Table S1 (supporting information).

\begin{tabular}{|c|c|c|c|c|}
\hline \multirow[b]{2}{*}{ Interaction } & \multicolumn{2}{|c|}{ HF/6-31G* } & \multicolumn{2}{|c|}{ Empirical $^{\text {b }}$} \\
\hline & $E_{\min }$ & $\boldsymbol{R}_{\min }$ & $E_{\min }$ & $\boldsymbol{R}_{\min }$ \\
\hline (a) $\mathrm{O}_{2 \mathrm{~A}}-\mathrm{H}_{\mathrm{w}}$ & -11.4 & 1.87 & -11.64 & 1.88 \\
\hline (b) $\mathrm{O}_{\mathrm{SA}}-\mathrm{H}_{\mathrm{w}}$ & -4.77 & 2.35 & -5.03 & 2.34 \\
\hline (c) $\mathrm{As}-\mathrm{O}_{\mathrm{w}}{ }^{\mathrm{c}}$ & -7.76 & 4.05 & 8.24 & 3.86 \\
\hline (d) $\mathrm{As}-\mathrm{O}_{\mathrm{w}}{ }^{\mathrm{d}}$ & -13.29 & 3.48 & -12.83 & 3.50 \\
\hline
\end{tabular}

Table 1. Empirical and HF/6-31G* water interaction energies and geometries for DMA ${ }^{\mathrm{a}}$.

${ }^{\mathrm{a}}$ Energies in $\mathrm{kcal} / \mathrm{mol}$ and distances in $\AA . R_{\min }$ is the water-DMA distance indicated by the dashed line shown in Figure 7; ${ }^{b}$ Empirical values were obtained from using the CHARMM36 optimized gas phase geometry; ${ }^{c}$ Interaction (c) has the plane of the water molecule perpendicular to plane of the $\mathrm{O}=\mathrm{As}=\mathrm{O}$ atoms;

${ }^{\mathrm{d}}$ Interaction (d) has the plane of the water molecule in the same plane as the $\mathrm{O}=\mathrm{As}=\mathrm{O}$ atoms.

The empirical potentials of stretching and bending motions were fitted into their corresponding potential energy surfaces of $\mathrm{HF} / 6-31 \mathrm{G}^{*}$ calculations. Furthermore, the force constants were re-optimized to the harmonic vibrational frequencies of scaled $\mathrm{HF} / 6-31 \mathrm{G}^{*}$ results and normal mode assignments (Table S2) using MOLVIB. The optimized stretching and bending parameters are provided in the Table S1. The next optimization focuses on the torsion parameters. The parameters of three torsion angles, $\mathrm{O}_{2 \mathrm{~A}}-\mathrm{As}-\mathrm{O}_{\mathrm{SA}}-\mathrm{C}_{\mathrm{T} 3}, \mathrm{O}_{\mathrm{SA}}-\mathrm{As}-\mathrm{O}_{\mathrm{SA}}-\mathrm{C}_{\mathrm{T} 3}$, and $\mathrm{As}-\mathrm{O}_{\mathrm{SA}}-\mathrm{C}_{\mathrm{T} 3}-\mathrm{H}_{\mathrm{A}}$, were optimized to fit the adiabatic torsional potential energy surface of $\mathrm{HF} / 6-31 \mathrm{G}^{*}$ results. Their torsional potential energy surfaces of 
empirical calculations and HF/6-31G* calculations were shown in Figure S1 (SI) and they are good agreement with each other. The optimized empirical data of these torsion angles are listed in Table S1 (SI).

\subsection{Molecular Dynamics Simulations}

We simulated the POAC and POPC lipid bilayers by molecular dynamics. The initial configurations of the POAC and POPC systems were prepared using the CHARMM-GUI developed by Im and co-workers [24]. The empirical parameters of the DMA developed above were used to model the arsenate group of POAC lipids, while other groups as well as counter ions were modeled using the CHARMM36 all-atom force field [25]. Water molecules were modeled using the TIP3 model [26]. Each lipid bilayer system consisted of 96 lipids (48 lipids for each leaflet), 4863 water molecules, $13 \mathrm{Na}^{+}$and $13 \mathrm{Cl}^{-}(0.15 \mathrm{M} \mathrm{NaCl})$; the corresponding water-lipid ratio was 50.63 . All MD simulations were performed using parallel NAMD $2.7 \mathrm{~b} 3$ software [27] with an NPT ensemble under three-dimensional periodic boundary conditions. The simulation temperature was controlled to $300 \mathrm{~K}$ through Langevin dynamics. The pressure was controlled at 1 bar using the Langevin piston Nosé-Hoover method [28] and the three orthogonal dimensions of the periodic box were allowed to vary independently. The van der Waals interactions were switched smoothly from 9.0 to $12.0 \AA$ to maintain energy stability. Cutoffs of 12.0 and $13.5 \AA$ were used to calculate the pair-wise interactions and generate the list of pairs, respectively. The non-bonded neighboring list was updated every 10 time steps. The particle-mesh Ewald technique [29] was used to treat the long-range electrostatic interactions. The hydrogen atom involved covalent bond lengths were constrained by the SHAKE algorithm [30], allowing the use of a larger integration time step of 2 fs. Prior to MD production runs, conjugate gradient energy minimization was performed to remove the bad contacts of the initial configuration. Next, a 0.1-ns slow heating simulation was performed. The molecular coordinates were stored every 5 ps. For all systems, a 150-ns MD simulation was performed. The trajectories of the final $75 \mathrm{~ns}$ simulation were used for statistical analysis. All analyses were performed by our in-house program, Pine-MD, which had been employed in previous MD studies of lipid bilayers [31], amyloidogenic peptides [32,33], and antimicrobial peptides [34]. Several structural and dynamics properties of lipid bilayers were calculated. The analysis methods are described in the Results and Discussion section.

\section{Conclusions and Summary}

Due to the chemical similarities of phosphorus and arsenic, the possibility of sustaining life if the phosphorus is substituted by arsenic has been suggested [1]. We simulated putative POAC lipid bilayers by all-atom molecular dynamics to understand its basic structural and dynamical properties. The POAC lipid is a putative lipid with the phosphorus of POPC lipid substituted by arsenic. We focus on understanding of the structural and dynamical properties of POAC lipid bilayers different from those of its analogous POPC lipid bilayers. Our simulations showed that the structural and dynamical properties of POAC lipid bilayers are distinct different from those of native POPC lipid bilayers. The POAC lipid bilayers have smaller lateral areas, greater thicknesses, and greater order than those of POPC lipid bilayers. Namely, the POPC lipid bilayers are more compact than that of POPC lipid bilayers. The compact structure of POAC lipid bilayers is due to the fact that POAC lipid bilayers form more inter-lipid arsenate-choline salt bridges than that of POPC lipid bilayers, which bind lipids 
together. Furthermore, these strong inter-lipid arsenate-choline interactions in POAC lipid bilayers also slow down the lipids' lateral diffusion and head group rotation. Since the POAC and POPC lipid bilayers have distinct structure and dynamics properties, it would be anticipated that POAC lipid bilayers will have different biological implications from native POPC lipid bilayers, e.g., the energy required for inter-cellular chemicals exchange.

\section{Acknowledgments}

We thank the National Science Council of Taiwan for financial support (grants 98-2113-M-008007-MY2 and 100-2113-M-008-003-MY2) and the National Center for High-Performance Computing and the V'ger computer cluster at the National Center University of Taiwan (contribution number: NCU-CCG102-0002) for computer time and facilities.

\section{References}

1. Wolfe-Simon, F.; Blum, S.J.; Kulp, T.R.; Gordon, G.W.; Hoeft, S.E.; Pett-Ridge, J.; Stolz, J.F.; Webb, S.M.; Weber, P.K.; Davies, P.C.; et al. A bacterium that can grow by using arsenic instead of phosphorus. Science 2011, 332, 1163-1166.

2. Fekry, M.I.; Tipton, P.A.; Gates, K.S. Kinetic consequences of replacing the internucleotide phosphorus atoms in DNA with arsenic. ACS Chem. Biol. 2011, 6, 127-130.

3. Wang, J.; Gu, J.; Leszczynski, J. Could hydrolysis of arsenic substituted DNA be prevented? Protection arises from stacking interactions. Chem. Commun. (Camb) 2012, 48, 3626-3628.

4. Reaves, M.L.; Sinha, S.; Rabinowitz, J.D.; Kruglyak, L.; Redfield, R.J. Absence of detectable arsenate in DNA from arsenate-grown GFAJ-1 cells. Science 2012, 337, 470-473.

5. Erb, T.J.; Kiefer, P.; Hattendorf, B.; Günther, D.; Vorholt, J.A. GFAJ-1 Is an Arsenate-Resistant, Phosphate-Dependent Organism. Science 2012, 337, 467-470.

6. Elias, M.; Wellner, A.; Goldin-Azulay, K.; Chabriere, E.; Vorholt, J.A.; Erb, T.J.; Tawfik, D.S. The molecular basis of phosphate discrimination in arsenate-rich environments. Nature 2012, 491, 134-137.

7. Denning, E.J.; Mackerell, A.D., Jr. Impact of arsenic/phosphorus substitution on the intrinsic conformational properties of the phosphodiester backbone of DNA investigated using ab initio quantum mechanical calculations. J. Am. Chem. Soc. 2011, 133, 5770-5772.

8. Xu, Y.; Ma, B.; Nussinov, R. Structural and functional consequences of phosphate-arsenate substitutions in selected nucleotides: DNA, RNA, and ATP. J. Phys. Chem. B 2012, 116, 4801-4811.

9. Rosen, B.P.; Ajees, A.A.; McDermott, T.R. Life and death with arsenic. Arsenic life: An analysis of the recent report "A bacterium that can grow by using arsenic instead of phosphorus". Bioessays 2011, 33, 350-357.

10. Stryer, L. Biochemistry, 3rd ed.; W.H. Freeman and Co.: New York, NY, USA, 1988.

11. Pabst, G.; Rappolt, M.; Amenitsch, H.; Laggner, P. Structural information from multilamellar liposomes at full hydration: Full q-range fitting with high quality X-ray data. Phys. Rev. E 2000, 62, 4000-4009. 
12. Smaby, J.M.; Momsen, M.M.; Brockman, H.L.; Brown, R.E. Phosphatidylcholine acyl unsaturation modulates the decrease in interfacial elasticity induced by cholesterol. Biophys. J. 1997, 73, 1492-1505.

13. Kučerka, N.; Tristram-Nagle, S.; Nagle, J. Structure of Fully Hydrated Fluid Phase Lipid Bilayers with Monounsaturated Chains. J. Membr. Biol. 2006, 208, 193-202.

14. Hyslop, P.A.; Morel, B.; Sauerheber, R.D. Organization and interaction of cholesterol and phosphatidylcholine in model bilayer membranes. Biochemistry 1990, 29, 1025-1038.

15. Poger, D.; Mark, A.E. On the validation of molecular dynamics simulations of saturated and cis-monounsaturated phosphatidylcholine lipid bilayers: A comparison with experiment. J. Chem. Theory Comput. 2009, 6, 325-336.

16. Poger, D.; Mark, A.E. Lipid bilayers: The effect of force field on ordering and dynamics. J. Chem. Theory Comput. 2012, 8, 4807-4817.

17. Seelig, J.; Waespe-Sarcevic, N. Molecular order in cis and trans unsaturated phospholipid bilayers. Biochemistry 1978, 17, 3310-3315.

18. Williams, G.; Watts, D.C. Non-Symmetrical dielectric relaxation behaviour arising from a simple empirical decay function. Trans. Faraday Soc. 1970, 66, 80-85.

19. Williams, G.; Watts, D.C.; Dev, S.B.; North, A.M. Further considerations of non symmetrical dielectric relaxation behaviour arising from a simple empirical decay function. Trans. Faraday Soc. 1971, 67, 1323-1335.

20. Mackerell, A.D.; Wiorkiewiczkuczera, J.; Karplus, M. An all-atom empirical energy function for the simulation of nucleic-acids. J. Am. Chem. Soc. 1995, 117, 11946-11975.

21. Frisch, M.J.; Trucks, G.W.; Schlegel, H.B.; Scuseria, G.E.; Robb, M.A.; Cheeseman, J.R.; Scalmani, G.; Barone, V.; Mennucci, B.; Petersson, G.A.; et al. Gaussian 09, Revision A.1; Gaussian, Inc.: Wallingford, CT, USA, 2009.

22. Brooks, B.R.; Brooks, C.L.; Mackerell, A.D.; Nilsson, L.; Petrella, R.J.; Roux, B.; Won, Y.; Archontis, G.; Bartels, C.; Boresch, S.; et al. CHARMM: The biomolecular simulation program. J. Comput. Chem. 2009, 30, 1545-1614.

23. Simon, S.L.; Duran, M.; Dannenberg, J.J. How does basis set superposition error change the potential surfaces for hydrogen-bonded dimers? J. Chem. Phys. 1996, 105, 11024.

24. Jo, S.; Kim, T.; Iyer, V.G.; Im, W. CHARMM-GUI: A web-based graphical user interface for CHARMM. J. Comput. Chem. 2008, 29, 1859-1865.

25. Klauda, J.B.; Venable, R.M.; Freites, J.A.; O’Connor, J.W.; Tobias, D.J.; Mondragon-Ramirez, C.; Vorobyov, I.; MacKerell, A.D.; Pastor, R.W. Update of the CHARMM All-Atom additive force field for lipids: Validation on six lipid types. J. Phys. Chem. B 2010, 114, 7830-7843.

26. Jorgensen, W.L.; Chandrasekhar, J.; Madura, J.D.; Impey, R.W.; Klein, M.L. Comparison of simple potential functions for simulating liquid water. J. Chem. Phys. 1983, 79, 926-935.

27. Kale, L.; Skeel, R.; Bhandarkar, M.; Brunner, R.; Gursoy, A.; Krawetz, N.; Phillips, J.; Shinozaki, A.; Varadarajan, K.; Schulten, K. NAMD2: Greater scalability for parallel molecular dynamics. J. Comp. Phys. 1999, 151, 283-312.

28. Feller, S.E.; Zhang, Y.; Pastor, R.W.; Brooks, B.R. Constant pressure molecular dynamics simulation: The Langevin piston method. J. Chem. Phys. 1995, 103, 4613-4621. 
29. Darden, T.; York, D.; Pedersen, L. Particle Mesh Ewald-An N.Log(N) Method for Ewald Sums in Large Systems. J. Chem. Phys. 1993, 98, 10089-10092.

30. Ryckaert, J.-P.; Ciccotti, G.; Berendsen, H.J.C. Numerical integration of the cartesian equations of motion of a system with constraints: Molecular dynamics of n-alkanes. J. Comp. Phys. 1977, 23, $327-341$.

31. Tsai, H.H.; Lai, W.X.; Lin, H.D.; Lee, J.B.; Juang, W.F.; Tseng, W.H. Molecular dynamics simulation of cation-phospholipid clustering in phospholipid bilayers: Possible role in stalk formation during membrane fusion. Biochim. Biophys. Acta 2012, 1818, 2742-2755.

32. Tsai, H.-H.G.; Lee, J.-B.; Tseng, S.-S.; Pan, X.-A.; Shih, Y.-C. Folding and membrane insertion of amyloid-beta (25-35) peptide and its mutants: Implications for aggregation and neurotoxicity. Proteins Struct. Funct. Bioinform. 2010, 78, 1909-1925.

33. Tsai, H.H.; Reches, M.; Tsai, C.J.; Gunasekaran, K.; Gazit, E.; Nussinov, R. Energy landscape of amyloidogenic peptide oligomerization by parallel-tempering molecular dynamics simulation: Significant role of Asn ladder. Proc. Natl. Acad. Sci. USA 2005, 102, 8174-8179.

34. Tsai, C.-W.; Hsu, N.-Y.; Wang, C.-H.; Lu, C.-Y.; Chang, Y.; Tsai, H.-H.G.; Ruaan, R.-C. Coupling molecular dynamics simulations with experiments for the rational design of indolicidin-analogous antimicrobial peptides. J. Mol. Biol. 2009, 392, 837-854.

(C) 2013 by the authors; licensee MDPI, Basel, Switzerland. This article is an open access article distributed under the terms and conditions of the Creative Commons Attribution license (http://creativecommons.org/licenses/by/3.0/). 